\title{
Presentación clínica de la apendicitis aguda en pacientes inmunocomprometidos por diabetes o $\mathrm{VIH} /$ sida
}

\author{
Guillermo Ponce de León-Ballesteros, Rafael Pérez-Soto, Karla Zúñiga-Posselt y \\ David Velázquez-Fernández
}

Secretaría de Salud, Instituto Nacional de Ciencias Médicas y Nutrición "Salvador Zubirán", Departamento de Cirugía, Ciudad de México, México

\begin{abstract}
Resumen
Introducción: Los pacientes inmunocomprometidos presentan respuesta inflamatoria limitada que puede retrasar el diagnóstico de la apendicitis aguda ( $A A)$. Objetivo: Evaluar si el inmunocompromiso puede afectar el curso clínico y evolución de la AA. Método: Análisis retrospectivo, comparativo, de pacientes sometidos a apendicectomía por $A A$ : con VIH, diabetes mellitus tipo 2 (DM2) y sin otra patología. Resultados: Se revisaron 128 pacientes con AA intervenidos quirúrgicamente (53.6\% del sexo femenino), edad media de 42.5 años, 15 (11.7 \%) tenían diagnóstico de VIH, 47 (36.7 \%) de DM2 y 66 (51.6 \%) no cursaban con otra enfermedad. La proporción de leucocitosis fue menor en el grupo con VIH (66.7 \%; $p=0.007)$. En los pacientes con VIH y DM2 se registró mayor tiempo de evolución: $66.9 \pm 61.2 y 90.1 \pm 144$ horas $(p \leq 0.001)$, mayor tiempo de estancia hospitalaria: $11.1 \pm 17.1$ y $6.5 \pm 4.1$ días ( $\leq \leq 0.0001$ ), mayor tasa de complicaciones: 20 y $23.8 \%(p=0.036)$. La complicación más frecuente fue la infección del sitio quirúrgico superficial y profunda. La hemicolectomía derecha fue más frecuente en el grupo con VIH $(20 \%, p=0.017)$. No se registró mortalidad. Conclusiones: La inmunodepresión afecta el curso clínico y evolución de la $A A$.
\end{abstract}

PALABRAS CLAVE: Apendicitis aguda. VIH. Diabetes mellitus. Inmunocompromiso. Complicaciones quirúrgicas.

\begin{abstract}
Introduction: Immunocompromised patients experience limited inflammatory response, which can delay acute appendicitis (AA) diagnosis. Objective: To assess if immunosuppression can affect $A A$ clinical course and evolution. Method: Comparative, retrospective analysis of patients with HIV or type 2 diabetes mellitus (DM2) or with no other pathology who underwent appendectomy for $A A$. Results: $A$ total of 128 patients with $A A$ who were surgically intervened were assessed (53.6\% were of the female gender); mean age was 42.5 years, 15 (11.7\%) had been diagnosed with HIV infection, $47(36.7 \%)$ with DM2 and $66(51.6 \%)$ had no other disease. The proportion of leukocytosis was lower in the HIV group (66.7\%; $p=0.007)$. Patients with HIV and DM2 had longer evolution time (HIV $66.9 \pm 61.2$, DM2 $90.1 \pm 144$ hours; $p \leq 0.001$ ), longer hospital length of stay (HIV 11.1 \pm 17.1, DM2 $6.5 \pm 4.1$ days; $p \leq 0.0001$ ), and a higher rate of complications (HIV 20\%, DM2 23.8\%; $p=0.036$ ). The most common complication was superficial and deep surgical site infection. Right hemicolectomy was more common in the HIV group (20\%; $p=0.017)$. There was no mortality registered. Conclusions: Immunosuppression affects AA clinical course and evolution.
\end{abstract}

KEY WORDS: Acute appendicitis. HIV. Diabetes mellitus. Immunocompromised. Surgical complications.

Correspondencia:

Guillermo Ponce de de León-Ballesteros

E-mail: guillermoplb@ hotmail.com
Fecha de recepción: 03-11-2017

Fecha de aceptación: 11-12-2017

DOI://dx.doi.org/10.24875/GMM.17003839
Gac Med Mex. 2018;154:473-479

Disponible en PubMed

www.gacetamedicademexico.com 


\section{Introducción}

La apendicitis aguda (AA) es la principal urgencia quirúrgica en el mundo, por lo que constituye en un problema de salud importante. La prevalencia de apendicitis aguda en la población general es de $0.1 \%$ y el riesgo de padecerla es de $8 \%$, mientras que en los pacientes con virus de inmunodeficiencia humana/síndrome de inmunodeficiencia adquirida (VIH/sida) la prevalencia es de 0.6 a $3.6 \% .^{1-3}$ Además, la incidencia de AA se ha incrementado en los países en desarrollo, probablemente debido al aumento de los casos de $\mathrm{VIH} /$ sida. $^{1}$ La mortalidad asociada con AA ha disminuido históricamente a partir de que se describió su tratamiento quirúrgico, de $26 \%$ a menos de 0.2 por cada 100000 habitantes en la actualidad. Sin embargo, la presencia de comorbilidades y de AA complicada o perforada puede aumentar la mortalidad hasta a $5 \%$. En los pacientes con $\mathrm{VIH} /$ sida con AA complicada llega a ser de $22 \%{ }^{1}$

Algunos reportes indican que la mayor incidencia de AA en los pacientes con $\mathrm{VIH} /$ sida podría relacionarse al hecho de que el apéndice vermiforme es un órgano blanco del virus. ${ }^{2}$ Asimismo, hasta $30 \%$ de los cuadros en dichos pacientes se relaciona con alguna comorbilidad, principalmente infecciosa, por ejemplo sarcoma de Kaposi, infección por citomegalovirus, Mycobacterium sp., Cryptosporidiosis y Salmonella typhi, entre otras. ${ }^{3}$

Los pacientes con $\mathrm{VIH} /$ sida con diagnóstico de $\mathrm{AA}$ en promedio tienen mayor edad que la población general. Algunas diferencias clínicas cuando se comparan estos pacientes con controles sin otra patología además de la AA son la menor frecuencia de leucocitosis ( $34.6 \%$ versus $87 \%$, respectivamente), menor frecuencia de fiebre $(46.1$ a $55 \%$ versus $66.5 \%$, respectivamente) .,3-5 $^{1,5}$

Se ha reportado mayor tasa de complicaciones perioperatorias en pacientes con $\mathrm{VIH} / \mathrm{sida}$, entre las más comunes infección del sitio quirúrgico, retraso o alteraciones en la cicatrización de la herida quirúrgica, mayor tasa de AA complicada (AA perforada, peritonitis generalizada, absceso intraabdominal), mayor tiempo de estancia intrahospitalaria y mayor mortalidad., ${ }^{1,-5}$

Los pacientes con $\mathrm{VIH} /$ sida presentan 15 veces más riesgo de apendicitis aguda perforada que la población general, siendo este el principal predictor de mortalidad. ${ }^{6}$

En cuanto a la diabetes mellitus tipo 2 (DM2), esta se asocia con mayor tasa de complicaciones. El mal control de la enfermedad se relaciona con la mayoría de las complicaciones. La glucosilación de las inmunoglobulinas es el principal mecanismo por el cual se encuentra afectado el sistema inmunológico, además de que existe alteración en la función de los polimorfonucleares, adherencia leucocitaria y disminución en la quimiotaxis y fagocitosis. ${ }^{7}$

Al igual que los pacientes con $\mathrm{VIH} /$ sida, los pacientes con DM2 con diagnóstico de AA tienen edad mayor que la población general, así como mayor tasa de complicaciones, de frecuencia de AA complicada y de estancia hospitalaria.

La mayor tasa de complicaciones puede estar asociada con el retraso del diagnóstico, alteraciones en el sistema inmunológico o la presencia de comorbilidades relacionadas o desencadenadas por el VIH/ sida y la DM2. ${ }^{7}$

La tasa de complicaciones es mayor en los grupos con $\mathrm{VIH} /$ sida y DM2 en comparación con la población general, como ya se mencionó. La tasa de AA complicada en los pacientes con $\mathrm{VIH} /$ sida es de 8 a $33 \%$ y en los pacientes con DM2 de 22.6 a $64.8 \%$, mientras que lo reportado para los pacientes sin otras enfermedades es de 3.5 a $19 \%{ }^{1,5,7,8}$ La peritonitis generalizada en los pacientes con $\mathrm{VIH} /$ sida se ha reportado hasta en $31 \%$, en los pacientes diabéticos en 18 a $22.6 \%$,-10 mientras que en la población general en 2 a $8 \%$, por lo que se concluye que estos grupos tienen hasta 15 veces más riesgo de presentar $A A$ complicada.

El objetivo de este estudio fue evaluar si el inmunocompromiso puede afectar el curso clínico y evolución de los pacientes con AA en relación con individuos sin otras enfermedades, además de comparar dos grupos diferentes de pacientes con inmunocompromiso y AA.

\section{Método}

Estudio observacional, retrospectivo, con alcance comparativo-correlacional. Fue llevado a cabo en el Instituto Nacional de Nutrición y Ciencias Médicas "Salvador Zubirán", centro de tercer nivel de atención en México. Se analizaron diversas variables: edad, sexo, síntomas y signos, diagnóstico clínico e histopatológico, resultados de laboratorio, hallazgos transoperatorios, técnica quirúrgica y complicaciones posoperatorias. Los pacientes fueron mayores de 18 años y se distribuyeron de la siguiente forma: grupo con $\mathrm{VIH}$ (15 pacientes), grupo con DM2 (47 pacientes) y grupo de pacientes aparentemente sin otra condición además de la $A A$ (66 controles) y en quienes no se tenía 


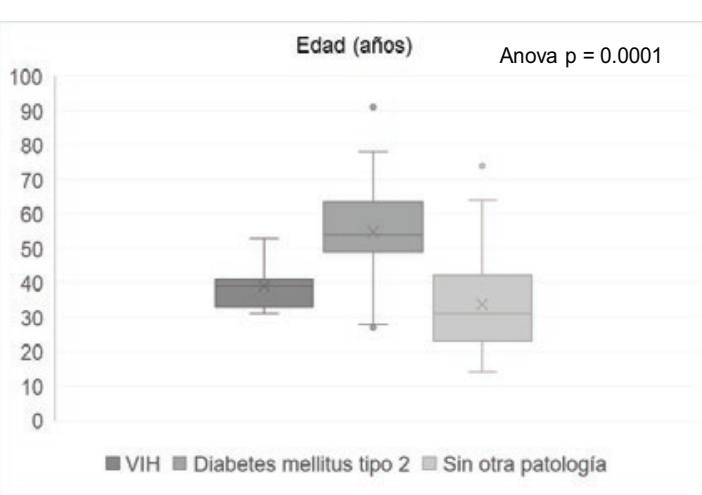

Figura 1. Edad promedio de los grupos.

evidencia de alguna enfermedad previa; todos fueron intervenidos quirúrgicamente por AA en el Instituto Nacional de Nutrición y Ciencias Médicas "Salvador Zubirán" entre 1990 y 2013. En los pacientes con VIH/ sida se analizaron otras variables como el tratamiento antirretroviral, clasificación de la enfermedad con base en la clasificación de la Organización Mundial de la Salud, carga viral y cuenta celular de linfocitos CD4+ al momento del diagnóstico de la AA.

Se incluyeron todos los pacientes en quienes se realizó apendicectomía abierta o laparoscópica con $\mathrm{VIH} /$ sida en el periodo mencionado; en cuanto a los pacientes control y con DM2, la muestra se obtuvo de forma probabilística y sistemática a partir de la lista de todos los pacientes intervenidos por AA en el mismo periodo (833 pacientes), con al menos una relación de 3:1 respecto a los pacientes con VIH. Se excluyeron los pacientes con diagnóstico de diabetes mellitus tipo 1 , neoplasias hematológicas o sólidas, enfermedades autoinmunes, bajo inmunosupresión farmacológica o con otro tipo de inmunocompromiso.

El análisis estadístico se realizó con los programas SPSS Statistics versión 20.0 y Excel. Se realizó análisis estadístico no paramétrico debido a la muestra recolectada. Para la comparación de las variables dimensionales se realizó prueba de $t$ de Student y Anova; para las ordinales y categóricas, prueba de tau de Kendall y chi cuadrado, respectivamente. Se consideró estadísticamente significativa una $p \leq 0.05$ o de $5 \%$ (error $\alpha$ ) para una prueba de hipótesis de dos colas.

El protocolo fue revisado y aprobado por el Comité Institucional de Investigación Biomédica en Humanos (referencia 1142).

\section{Resultados}

De los 128 pacientes, 67 eran hombres (53.6\%) y 58 mujeres $(46.4 \%)$, con edad de $42.5 \pm 15.8$ años, rango de 18 a 91; 15 (11.7\%) tenían diagnóstico de $\mathrm{VIH}$, $47(36.7 \%)$ de DM2 y 66 (51.6 \%) fueron controles. El grupo control fue significativamente más joven (33.67 años versus 39 años de los pacientes con $\mathrm{VIH}$ versus 54.74 años en aquellos con DM2, $p \leq 0.001$ ) (Figura 1). Del grupo de pacientes con $\mathrm{VIH} 13 / 15(86.6 \%$, $p=0.007$ ) eran hombres, mientras que 19/47 (40.4\%) en el grupo con DM2 y $36 / 66$ (54.5\%) en el control.

Se encontró que todos los pacientes con VIH estaban recibiendo terapia antirretroviral. En cuanto a las enfermedades asociadas con $\mathrm{VIH} /$ sida, un paciente tenía diagnóstico reciente de infección diseminada por citomegalovirus y tres, antecedente de tuberculosis pulmonar, sin embargo, en ninguno se pudo determinar si la AA estaba relacionada con estos diagnósticos. En uno, el reporte histopatológico definitivo fue tumor carcinoide de apéndice y en el resto, AA. Los pacientes con menor cuenta de linfocitos CD4+ tuvieron menor frecuencia de leucocitosis comparados con los demás pacientes del grupo, sin significación estadística (Anova $\mathrm{p}=0.47$ ). Los pacientes que no presentaron leucocitosis fueron catalogados conforme a la clasificación de la Organización Mundial de la Salud en estadio 3 por sus cuentas actuales o previas de linfocitos CD4+. Una carga viral detectable ni el número de copias estuvieron correlacionados con algún factor pronóstico o complicación (chi cuadrado $\mathrm{p}=0.31$ ) (Tabla 1).

Las variantes demográficas, así como los datos clínicos y perioperatorios en los diversos grupos se describen las Tablas 2 y 3 . La proporción de leucocitosis fue menor en el grupo con $\mathrm{VIH}: 66.7 \%$ versus $85.1 \%$ en el grupo con DM2 y $85.7 \%$ en el grupo control (chi cuadrado $p=0.007$ ), sin embargo, no hubo diferencia entre el grupo con DM2 y el control $(p=0.9)$ (Figura 2).

En cuanto a la presentación clínica, una menor proporción de los pacientes con VIH presentaron dolor abdominal difuso: $60 \%$ versus $83 \%$ del grupo con DM2 y $89 \%$ del control (chi cuadrado, $p=0.022$ ), así como de dolor en la fosa iliaca derecha: $80 \%$ versus $98 \%$ del grupo con DM2 y $94 \%$ del control (chi cuadrado, $p=0.046$ ). El tiempo de evolución fue superior en los pacientes con VIH y DM2 respecto a los controles: $66.9 \pm 61.2,90.1 \pm 144$ y28 \pm 22.6 horas, respectivamente (Anova $p \leq 0.001$ ) (Figura 3). Una mayor proporción de pacientes con VIH y DM2 tuvieron fiebre en relación con los controles: 20, 27.7 y $11.3 \%$, respectivamente (chi cuadrada $p=0.09$ ), $\sin$ alcanzar significancia.

La presentación de AA complicada fue mayor en el grupo con DM2: $50 \%$ versus $26.7 \%$ grupo con $\mathrm{VIH}$ y $27.3 \%$ en el grupo control (chi cuadrado $p=0.004$ ), 
Tabla 1. Características de los pacientes con VIH y apendicitis aguda

\begin{tabular}{|c|c|c|c|c|c|c|c|c|c|c|}
\hline Paciente & $\begin{array}{l}\text { Clasificación } \\
\text { OMS }\end{array}$ & $\begin{array}{l}\text { Carga } \\
\text { viral }\end{array}$ & CD4+ & $\begin{array}{l}\text { Leucocitos } \\
\text { previos } \\
\text { AA }\end{array}$ & $\begin{array}{c}\text { Leucocitos } \\
\text { AA }\end{array}$ & Comorbilidades & $\begin{array}{l}\text { Hallazgo } \\
\text { transoperatorio }\end{array}$ & Hemicolectomía & $\begin{array}{c}\text { Estancia } \\
\text { hospitalaria } \\
\text { (días) }\end{array}$ & Complicaciones \\
\hline 1 & A2 & - & 366 & ND & 19600 & - & $\begin{array}{l}\text { Absceso } \\
\text { intraabdominal }\end{array}$ & Sí & 4 & $\begin{array}{l}\text { Sangrado } \\
>500 \mathrm{~mL}\end{array}$ \\
\hline 2 & A3 & - & 577 & 6800 & 18200 & - & AA & - & 2 & \\
\hline 3 & A1 & - & 761 & 6200 & 14500 & - & AA & - & 2 & \\
\hline 4 & A3 & - & 337 & 5300 & 8600 & CMV & AA & - & 2 & \\
\hline 5 & A3 & - & 180 & 4600 & 10900 & - & $\begin{array}{l}\text { Apéndice } \\
\text { perforado }\end{array}$ & - & 9 & $I S Q$ \\
\hline 6 & A2 & 14700 & 283 & 3900 & 11100 & MTb & AA & - & 5 & \\
\hline 7 & C3 & - & 65 & 2100 & 3300 & - & AA & - & 67 & \\
\hline 8 & A2 & - & 633 & 7800 & 12200 & - & AA & - & 5 & \\
\hline 9 & C3 & - & 565 & ND & 17500 & - & AA & - & 2 & \\
\hline 10 & A2 & - & 764 & 7000 & 19730 & - & $\begin{array}{l}\text { Absceso } \\
\text { intraabdominal }\end{array}$ & - & 30 & $\begin{array}{l}\text { ISQ } \\
\text { Reoperación }\end{array}$ \\
\hline 11 & A2 & 51627 & 308 & 5100 & 18700 & - & AA & - & 3 & \\
\hline 12 & C3 & - & 423 & 5000 & 14800 & - & AA & - & 3 & \\
\hline 13 & C3 & 78 & 441 & 5200 & 6500 & - & $\begin{array}{l}\text { AA } \\
\text { Tumor } \\
\text { carcionoide }\end{array}$ & Sí & 13 & ISQ \\
\hline 14 & C3 & - & 329 & 5200 & 8000 & MTb & $\begin{array}{l}\text { Absceso } \\
\text { intraabdominal }\end{array}$ & Sí & 6 & $\begin{array}{l}\text { Sangrado } \\
1200 \mathrm{~mL}\end{array}$ \\
\hline 15 & C3 & 2500 & 99 & 3200 & 2100 & MTb & $\mathrm{AA}$ & - & 13 & \\
\hline
\end{tabular}

AA = Apendicitis aguda, $\mathrm{CMV}=$ Citomegalovirus, $\mathrm{MTb}=$ Mycobacterium tuberculosis, ISQ = Infección de sitio quirúrgico

así como la presencia de absceso intraabdominal: $39.1 \%$ versus $20 \%$ en el grupo con $\mathrm{VIH}$ y $9 \%$ en el control (chi cuadrado $p=0.001$ ), sin embargo, la $A A$ perforada no demostró diferencia entre los grupos, pese a ser de mayor frecuencia en el grupo control: $18.2 \%$ versus $6.7 \%$ en el grupo con $\mathrm{VIH}$ y $10.9 \%$ en el grupo con DM2 (chi cuadrado $p=0.37$ ) (Figura 4). Fueron catalogados con AA complicada $26 \%$ de los pacientes con $\mathrm{VIH}$ : tres pacientes con absceso intraabdominal y uno con AA perforada.

El abordaje laparoscópico fue empleado con mayor frecuencia en el grupo control, con una diferencia significativa: $36.4 \%$ versus $13.3 \%$ en el grupo con $\mathrm{VIH}$ y $13 \%$ en el grupo con DM2, chi cuadrado $\mathrm{p}=0.011)$. En un paciente del grupo con $\mathrm{VIH}(6.7 \%)$, el abordaje laparoscópico tuvo que convertirse a apendicectomía abierta, en cinco (10.9\%) del grupo con DM2 y en dos (3\%) de los controles (chi cuadrado $p=0.24$ ). En la Figura 5 se muestran los porcentajes del tipo de abordaje empleado en los grupos. La necesidad de realizar hemicolectomía derecha fue mayor para el grupo con $\mathrm{VIH}$, respecto a los otros grupos: $20 \%$ versus $6.3 \%$ en grupo con DM2 y $1.5 \%$ en grupo control (chi cuadrado $p=0.017$ ).

La indicación de hemicolectomía derecha en dos pacientes $(13.3 \%)$ del grupo con VIH fue por absceso intraabdominal y afección de la base del apéndice vermiforme y en uno (6.6\%) por sospecha de tumor, en el cual el diagnóstico histopatológico fue tumor carcinoide apendicular. La hemicolectomía derecha se realizó por plastrón abscedado en el grupo con DM2, mientras que en el único paciente que la requirió en el grupo control fue por afección importante del ciego. El tiempo quirúrgico también fue significativamente mayor para el grupo con $\mathrm{VIH}: 112.9$ minutos versus 99.4 en el grupo con DM2 y 77.7 en el control (Anova $p=0.037$ ) (Figura 6), así como el sangrado intraoperatorio, con significación estadística: $214.2 \pm 364.4 \mathrm{~mL}$ versus $162.02 \pm 254.9$ y $33 \pm 29.3 \mathrm{~mL}$ en los grupos con DM2 y control, respectivamente (Anova $p=0.001$ ).

La proporción de complicaciones posoperatorias fue mayor en los grupos con VIH y DM2: 20 y $23.8 \%$, 
Tabla 2. Descripción de las características demográficas y clínicas de pacientes con apendicitis aguda

\begin{tabular}{lcccc}
\hline Característica & $\begin{array}{c}\text { Grupo 1 } \\
\text { VIH/sida }\end{array}$ & $\begin{array}{c}\text { Grupo 2 } \\
\text { DM2 }\end{array}$ & $\begin{array}{c}\text { Grupo 3 } \\
\text { Control }\end{array}$ & p \\
\hline Edad (años) & 39 & 54.74 & 33.67 & $\leq 0.001$ \\
Leucocitosis (\%) & 66.7 & 85.1 & 85.7 & 0.007 \\
Fiebre (\%) & 20 & 27.7 & 11.3 & 0.09 \\
Tiempo de & 66.87 & 90.07 & 28 & 0.001 \\
evolución (horas) & & & & \\
Dolor en FID (\%) & 80 & 98 & 94 & 0.046 \\
Dolor abdominal & 60 & 83 & 89 & 0.022 \\
difuso (\%) & & & & \\
Rebote (\%) & 73 & 83 & 77 & 0.64 \\
Migración del & 60 & 68 & 75 & 0.43 \\
dolor (\%) & & & & \\
Nausea (\%) & 80 & 74 & 86 & 0.3 \\
Vomito (\%) & 60 & 45 & 62 & 0.19 \\
Hiporexia (\%) & 100 & 81 & 84 & 0.19 \\
Escala & 7.13 & 7.87 & 7.63 & 0.32 \\
Alvarado (media) & & & & \\
\hline
\end{tabular}

Tabla 3. Descripción de las características perioperatorias de pacientes con apendicitis aguda

\begin{tabular}{lllll}
\hline Característica & $\begin{array}{l}\text { Grupo 1 } \\
\text { VIH/sida }\end{array}$ & $\begin{array}{l}\text { Grupo 2 } \\
\text { DM2 }\end{array}$ & $\begin{array}{l}\text { Grupo 3 } \\
\text { Sanos }\end{array}$ & p \\
\hline AA no complicada (\%) & 73.3 & 50 & 72.7 & 0.035 \\
AA perforada (\%) & 6.7 & 10.9 & 18.2 & 0.37 \\
$\begin{array}{l}\text { Absceso } \\
\text { intraabdominal (\%) }\end{array}$ & 20 & 39.1 & 9 & 0.001 \\
$\begin{array}{l}\text { Tiempo } \\
\text { quirúrgico (minutos) }\end{array}$ & 112.86 & 99.37 & 77.7 & 0.037 \\
$\begin{array}{l}\text { Hemicolectomía } \\
\text { derecha (\%) }\end{array}$ & 20 & 6.3 & 1.5 & 0.016 \\
Sangrado (mL) & 214.17 & 162.02 & 33 & 0.001 \\
Transfusión (\%) & 20 & 6.7 & 2 & 0.021 \\
Reoperación (\%) & 6.7 & 0 & 0 & 0.025 \\
$\begin{array}{l}\text { Estancia } \\
\text { hospitalaria (días) }\end{array}$ & 11.07 & 6.47 & 3.3 & $\leq 0.0001$ \\
Complicaciones (\%) & 20 & 23.8 & 4.8 & 0.036 \\
\hline
\end{tabular}

respectivamente, versus $4.8 \%$ en el grupo control (chi cuadrado $p=0.036$ ). Todos los pacientes del grupo con VIH presentaron una infección del sitio quirúrgico superficial; en el grupo con DM2, cuatro (8.6\%) presentaron absceso intraabdominal y siete (14.9\%), infección del sitio quirúrgico superficial. En el grupo control, dos

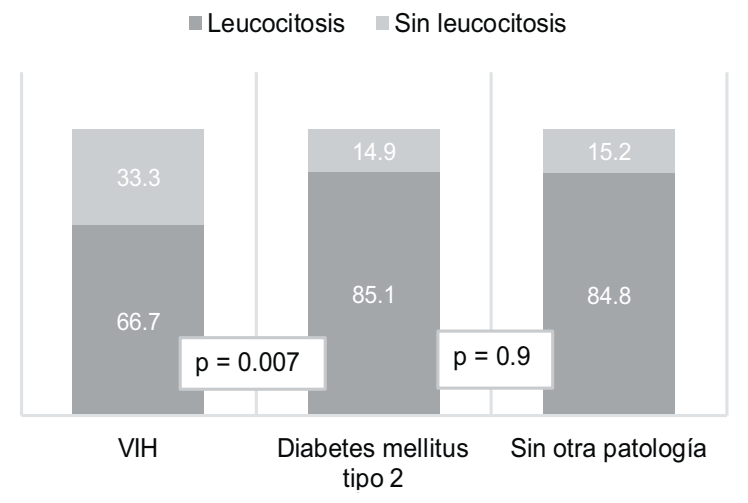

Figura 2. Proporción de leucocitosis por grupo. Chi cuadrado al comparar el grupo control con los grupos con VIH y diabetes mellitus tipo 2.

pacientes (3\%) presentaron infección del sitio quirúrgico superficial y uno (1.5\%), absceso intraabdominal. Los grupos con VIH y DM2 tuvieron mayor tiempo de estancia hospitalaria: $11.1 \pm 17.1$ y $6.5 \pm 4.1$ días, respectivamente, versus $3.2 \pm 4.1$ días en el grupo control (Anova $p \leq 0.0001$ ). No hubo mortalidad en ningún grupo. El análisis post-hoc comparando el porcentaje de complicaciones en los grupos con VIH y DM2 mostró poder $\beta$ de $83.9 \%$ y error $\alpha$ de 0.05 .

\section{Discusión}

Nuestro estudio demostró que los pacientes con VIH y DM2 tuvieron mayor edad, lo que concuerda con lo descrito en otros estudios., ${ }^{1,7}$

En cuanto a la presentación clínica, los dos grupos estudiados presentaron mayor tiempo de evolución; los pacientes con VIH, menor proporción de dolor abdominal difuso y en la fosa iliaca derecha y menor frecuencia de leucocitosis (66.7\%). En los estudios previos en los que se compararon pacientes con $\mathrm{VIH} /$ sida y pacientes sin otra patología además de $A A$, estos últimos presentaron con mayor frecuencia leucocitosis: $87 \%$ versus 34.6 y $88 \%$, así como fiebre: $66.5 \%$ versus 46.1 y $55 \%$. ${ }^{14,5}$ En nuestra serie, los pacientes con VIH tuvieron mayor proporción de fiebre que el grupo control, sin ser estadísticamente significativa. Otros datos clínicos como la migración del dolor, anorexia, náusea, vómito y rebote no fueron significativas en cuanto a la frecuencia de presentación, tal como reportaron Gliti et al. ${ }^{1}$

En los pacientes con DM2 se observaron mayor proporción de AA complicada (50\%), mientras que en el grupo con VIH el porcentaje fue similar al del grupo control (25.6 y $27.3 \%$, respectivamente), sin embargo, 


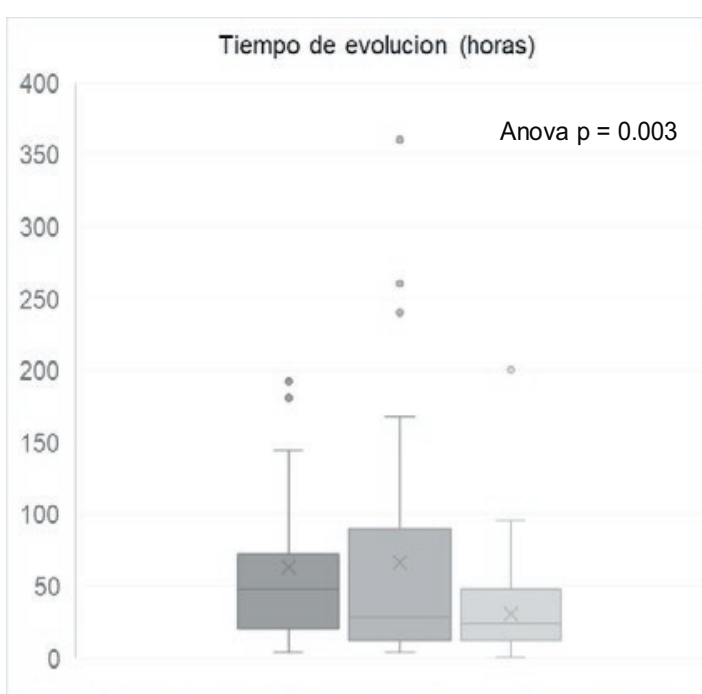

घ VIH $\square$ Diabetes mellitus tipo $2 \square$ Sin otra patología

Figura 3. Tiempo de evolución del cuadro clínico por grupo.

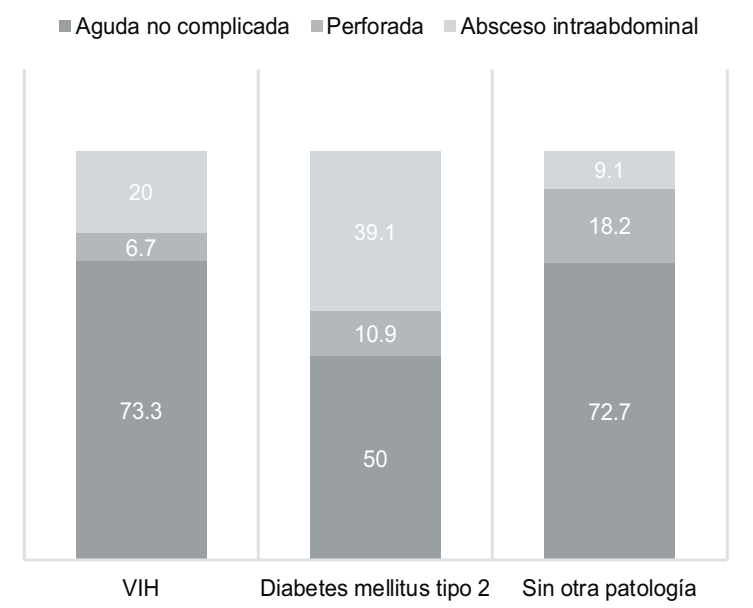

Figura 4. Proporción de apendicitis aguda no complicada y complicada (perforada/absceso intraabdominal) por grupo.

un porcentaje mayor de pacientes con $\mathrm{VIH}$ requirieron hemicolectomía derecha (20\%). La alta frecuencia de AA complicada en el grupo control no concordó con lo reportado en otras series, probablemente debido a que el lugar donde se llevó a cabo el estudio es un centro de tercer nivel y los pacientes suelen presentar complicaciones. De los pacientes con DM2 que presentaron AA complicada, $39.1 \%$ fue por absceso intraabdominal y $10.9 \%$ AA por perforación, mientras que $20 \%$ de los pacientes con VIH presentaron absceso intraabdominal y $5.6 \%$ AA perforada.

En 2008, Tsai et al. compararon a 71 pacientes con DM2 que desarrollaron AA versus pacientes sin DM2 y $A A$; los primeros tuvieron mayor riesgo de complicaciones relacionadas con la $A A$, mayor tiempo de estancia ( $11 \pm 1.1$ días versus $4.4 \pm 0.1$ días $\mathrm{p}=0.001)$ y mayor proporción de AA complicada $(64.8 \%)$. La mayor tasa de complicaciones puede estar en relación con el retraso del diagnóstico, alteraciones en el sistema inmunológico o la presencia de comorbilidades relacionadas con DM2 (enfermedad micro 0 macrovascular y nefropatía). ${ }^{7}$ Nuestros hallazgos en los pacientes con VIH correlacionaron con los encontrados en otras series, en las cuales se ha reportado una tasa de AA complicada de 8 a $33 \%$, incluso se ha reportado un riesgo 15 veces mayor de AA perforada que en la población general. ${ }^{1,5,8}$

Los pacientes con VIH y DM2 mostraron más complicaciones posoperatorias (infección del sitio quirúrgico superficial, absceso intraabdominal), así como mayor tiempo operatorio y de estancia hospitalaria. Se ha reportado que hasta $11.5 \%$ de los pacientes con VIH presentan infección del sitio quirúrgico, más que la población general, en la cual es de 2 a $4 \%$; en nuestra serie fue de $20 \% .{ }^{1}$ En los pacientes diabéticos también se ha descrito mayor proporción de infección del sitio quirúrgico: Sivrikos et al. registraron $6.1 \%$; en nuestro grupo con DM2 fue de 14.9 y $8.6 \%$ de infección a órganos y espacios. ${ }^{10}$

En cuanto al tiempo de estancia hospitalaria, en los pacientes con VIH se ha reportado un promedio de 4.4 a 9.3 días, mayor que en la población general. ${ }^{4,5}$ No es diferente para los pacientes con DM2 en quienes se describe de 2.5 a 11 días, además es mayor para los casos de AA complicada (4 a 11 días). ${ }^{7,10}$ En nuestra serie, la estancia hospitalaria para los grupos con VIH/sida, DM2 y control fue de $11,6.47$ y 3.24 días, respectivamente, similar a la descrita en la literatura.

Nuestro estudio tuvo diversas limitaciones debido a que fue retrospectivo y se realizó en un solo centro y con una muestra pequeña de pacientes con $\mathrm{VIH}$, sin embargo, en el análisis post-hoc comparando el porcentaje de complicaciones obtenidas en los grupos con VIH y DM2 su obtuvo un poder $\beta$ de $83.9 \%$ con un error $\alpha$ de 0.05 .

\section{Conclusiones}

Los pacientes con $\mathrm{VIH}$ o DM2 con AA mostraron mayor tiempo de evolución, lo que implicó retraso en el diagnóstico, mayor frecuencia de complicaciones posoperatorias y mayor tiempo de estancia hospitalaria en comparación con los sujetos del grupo control. La complicación más frecuente fue la infección del sitio quirúrgico. La inmunodepresión afecta el 


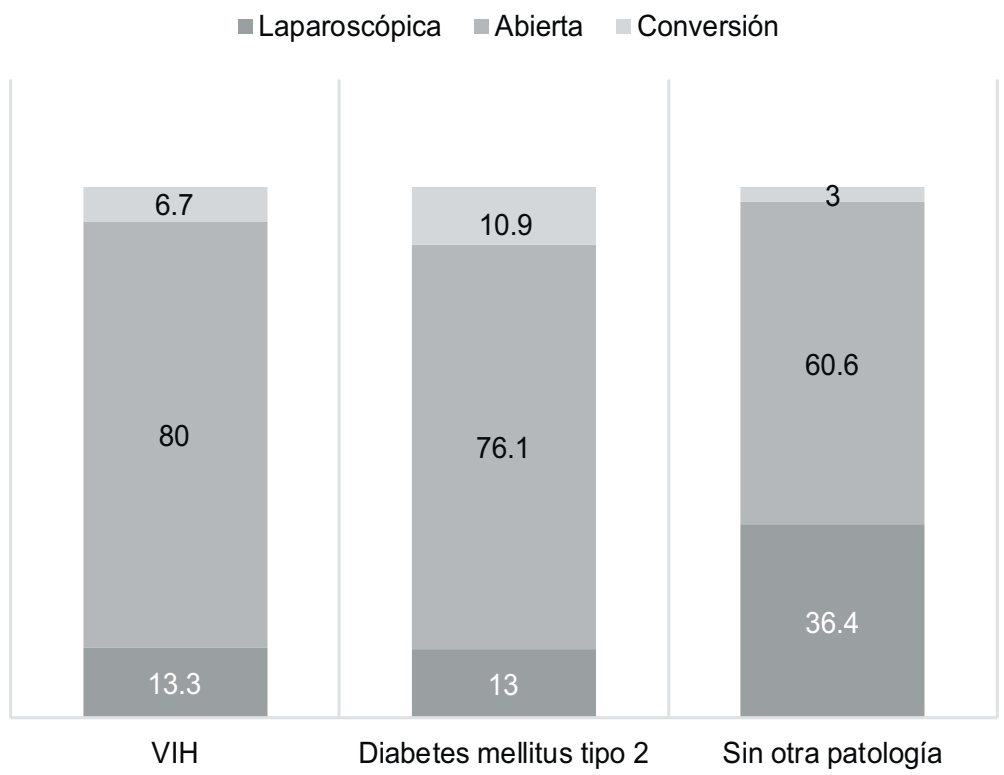

Figura 5. Proporción de los abordajes quirúrgicos empleados y conversión por grupo.

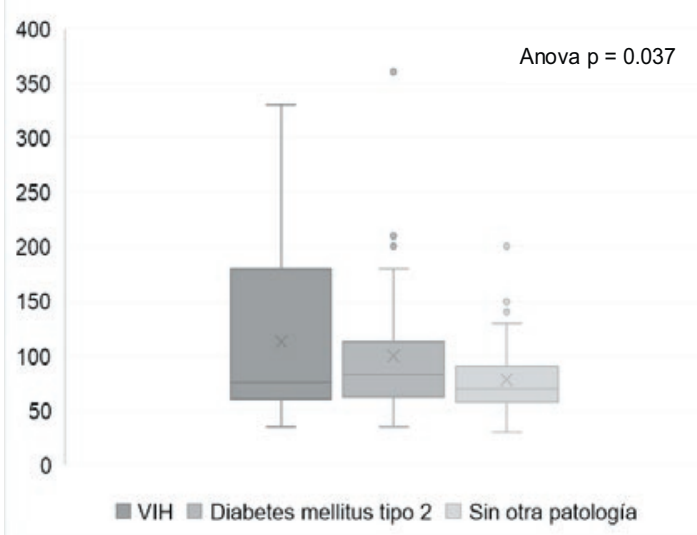

Figura 6. Tiempo quirúrgico en minutos por grupos.

curso clínico y evolución de los pacientes con AA. La evaluación cuidadosa y temprana y el tratamiento quirúrgico oportuno pueden abatir la mayor frecuencia de complicaciones.

\section{Bibliografía}

1. Giiti G, Mazigo H, Heukelbach J, Mahalu W. HIV, appendectomy and postoperative complications at a reference hospital in Northwest Tanzania: cross-sectional study. AIDS Res Ther. 2010;7:47.

2. Mueller GP, Williams RA. Surgical infections in AIDS patients. Am J Surg. 1995;169:34S-38S.

3. Crum-Cianflone N, Weekes J, Bavaro M. Appendicitis in HIV-infected patients during the era of highly active antiretroviral therapy. HIV Med. 2008;9:421-426.

4. Whitney TM, Macho JR, Russell TR, Bossart KJ, Heer FW, Schecter WP. Appendicitis in acquired inmmunodeficiency syndrome. Am J Surg. 1992:164:467-470.

5. Liu K, Shyu J, Uen YH, Chen TH, Shyr YM, Su CH, et al. Acute apendicitis in patients with acquired immunodeficiency syndrome. J Chin Med Assoc. 2005;68:226-229.

6. Cooperman M. Complication of appendectomy. Surg Clin North Am. 1983;63:1233-1247.

7. Tsai S, Hsu C, Chen S, Lin YY, Chu SJ. Complicated acute appendicitis in diabetic patients. Am J Surg. 2008;196:34-39.

8. Salzman DJ, Williams RA, Gelfand DV, Wilson SE. The surgeon and AIDS: twenty years later. Arch Surg. 2005;140:961-967.

9. Aldeen T, Horgan M, Macallan DC, Thomas V, Hay P. Is acute appendicitis another inflammatory condition associated with highly active antiretroviral therapy (HAART)? HIV Med. 2000;10:252-255.

10. Sivrikoz E, Karamanos E, Beale E, Teixeira P, Inaba K, Demetriades D. The effect of diabetes on outcomes following emergency appendectomy in patients without comorbidities: a propensity score-matched analysis of National Surgical Quality Improvement Program database. Am J Surg. 2015;209:206-211. 